\title{
Hot slumping glass technology and integration process to manufacture a grazing incidence scaled prototype for the IXO telescope modules
}

\author{
M. Ghigo ${ }^{a}$, S. Basso ${ }^{a}$, R. Canestrari ${ }^{\mathrm{a}, \mathrm{b}}$,, P. Conconi ${ }^{\mathrm{a}}$, O. Citterio ${ }^{\mathrm{a}}$, M. Civitani ${ }^{\mathrm{a}, \mathrm{b}}$, E. Dell'Orto $^{\mathrm{c}}$, \\ D. Gallieni ${ }^{\mathrm{d}}$, G. Pareschi ${ }^{\mathrm{a}}$, G. Parodi ${ }^{\mathrm{e}}$, L. Proserpio ${ }^{\mathrm{a}, \mathrm{b}}$, D. Spiga ${ }^{\mathrm{a}}$ \\ ${ }^{a}$ INAF-OAB Astronomical Observatory of Brera, Via E. Bianchi 46, 23807 Merate -LC- Italy \\ ${ }^{\mathrm{b}}$ Insubria University, Via Valleggio 11, Como -CO- Italy \\ 'Università Milano Bicocca, Milano -MI- Italy \\ ${ }^{d}$ A.D.S. Srl, Via Roma 87, 23868 Valmadrera -LC- Italy \\ ${ }^{e}$ BCV Progetti, Via S. Orsola 1, 20123 Milano - Italy
}

\begin{abstract}
As for all space missions, the limit imposed on the payload mass budget by the launcher is the main driver that forces the use of very lightweight optics. Considering the International X-ray Observatory (IXO) mission the present configuration has a mirror collecting area in the order of $3 \mathrm{~m}^{2}$ at $1.25 \mathrm{keV}, 0.65 \mathrm{~m}^{2}$ at $6 \mathrm{keV}$, and $150 \mathrm{~cm}^{2}$ at $30 \mathrm{keV}$. These large collecting areas could be obtained with a mirror assembly composed of a large number of high quality glass segments each being able to deliver the required angular resolution better or equal to 5 arcsec. These segments will form a X-Ray Optical Unit (XOU), an optical subunit of the telescope, and the XOUs will be assembled to form the whole mirror system. Based on the INAF-OAB experience in the thermal slumping of thin glass optics, a possible approach for the realization of large size and lightweight $\mathrm{X}$-ray mirrors is described in this paper.
\end{abstract}

Moulds made in a suitable material (as for example Silicon Carbide or Fused Silica) and having the suitable (parabolic and hyperbolic) profile are used for the realization of thin glass Mirror Plates (MP), with dimensions in the range of 200$400 \mathrm{~mm}$. After a thermal cycle the slumped MPs are characterized for acceptation and handled by means of an active support using vacuum suction for the integration phase. In this phase an active optical feedback is used to ensure the correct alignment of the MPs within the XOU. The MPs are then glued in its proper position in the XOU using also suitable glass ribs for the stiffening of the whole module.

An investigation in the problems and possible solutions to the slumping, measurement, integration and testing of the glass MPs into the XOU will be given, particularly with respect to a XOU scaled prototype that will be manufactured and used to assess the technology.

Keywords: Hot slumping, IXO telescope, XOU x-ray optical unit, Glass Mirror Plate

\section{INTRODUCTION}

In July 2008, the International X-ray observatory (IXO) was announced to the astronomical community. IXO is a joint Xray observatory with participation from ESA, NASA and JAXA. This mission supersedes the XEUS and Constellation-X mission concepts previously studied by ESA and NASA respectively.

IXO is dedicated to deep imaging and high resolution x-ray spectroscopy, with 100 times the throughput of previous Xray observatories (i.e. XMM Newton and Chandra). The main goal is the study of high energy universe phenomena in the X-ray band, which characterize the evolution of cosmic structures on both large and small scales; IXO will give scientist the possibility to draw conclusions on matter and energy under extreme conditions, formation and evolution of black holes, galaxies and clusters.

To meet these scientific goals, IXO has to verify some demanding requirements, mainly with respect to the optical payload whose required parameters are reported in Table 1. In particular, the large effective area is 50 times larger than Chandra and eight times larger than XMM-Newton, and the long focal length $(20 \mathrm{~m})$ pose a big challenge to fit the satellite and its payload in the launcher fairing, respecting dimensions and weight constraints.

Optics for EUV, X-Ray, and Gamma-Ray Astronomy IV, edited by Stephen L. O'Dell,

Giovanni Pareschi, Proc. of SPIE Vol. 7437, 74370P · (c) 2009 SPIE

CCC code: $0277-786 X / 09 / \$ 18 \cdot$ doi: $10.1117 / 12.826388$

Proc. of SPIE Vol. 7437 74370P-1 
The current baseline for IXO features a single large x-ray mirror with a $20 \mathrm{~m}$ focal length met using an extensible optical bench. Five focal plane instruments are interchangeable in the focus of the telescope: a wide field imaging and a hard Xray imaging detector, a high-spectral-resolution imaging spectrometer (calorimeter), a grating spectrometer, a high timing resolution spectrometer, and a polarimeter.

\begin{tabular}{|l|l|}
\hline Geometry & $\begin{array}{l}\text { Wolter I or Double-conical } \\
\text { approximation to Wolter I }\end{array}$ \\
\hline Focal lenght & $20 \mathrm{~m}$ \\
\hline Field of view & 18 arcmin diameter \\
\hline Coating & Ir + C overcoating \\
\hline Radius of clear aperture & $0.25-1.90 \mathrm{~m}$ \\
\hline Total mirror assembly mass (including & $1800 \mathrm{~kg}(+20 \%$ margin) \\
structure) & $3.00 \mathrm{~m}^{2} @ 1.25 \mathrm{keV}$ \\
\hline Effective area & $0.65 \mathrm{~m}^{2} @ 6.00 \mathrm{keV}$ \\
& $150 \mathrm{~cm}^{2} @ 30.00 \mathrm{keV}$ \\
\hline Angular resolution & $5 \operatorname{arcsec} \mathrm{HPD}, 0.1-7 \mathrm{keV}$ \\
& $30 \operatorname{arcsec} \mathrm{HPD}, 7.0-40 \mathrm{keV}$ \\
\hline
\end{tabular}

Table 1. Main requirements for IXO telescope coming from the scientific goals

The realization of the mirror unit appeared from the beginning as one of the technology driver. For such a big size, it is impossible to rely on monolithic pseudo-cylindrical close mirror shells, losing the intrinsic stiffness coming from a close structure with cylindrical symmetry. Due to the IXO mirror maximum aperture dimensions, the shells need to be azimuthally divided into segments with dimensions compatible with the technology selected to produce them and with the required stiffness to be mounted in the mirror structure (or substructure). Moreover, due to the mass limits imposed by the launcher capabilities, IXO relies on an area-to-mass ratio much higher than those of operating observatories: IXO requires achieving a value of about $20 \mathrm{~cm}^{2} / \mathrm{kg}$ versus $0.7 \mathrm{~cm}^{2} / \mathrm{kg}$ of Chandra and $4 \mathrm{~cm}^{2} / \mathrm{kg}$ of XMM Newton. The goal of having an angular resolution of 5 arcsec HPD for energies up to $7 \mathrm{keV}$ represents a challenge, too. Finally, taking into account the huge reflecting surface of the optics, the mirror manufacturing process should be scalable at high volume production industrial level at affordable financial costs and in an accessible time.

For all the mentioned reasons, the technologies used for the realization of operating observatories cannot be applied for the manufacturing of IXO mirrors: the direct polishing of each glass shell adopted by Chandra can not be afforded in terms of costs and production time; the replica process by electroformed Ni used for XMM Newton does not allow to meet the requirement in terms of mass due to the relatively high specific weight of $\mathrm{Ni}\left(8.8 \mathrm{~g} / \mathrm{cm}^{3}\right)$. The need to explore new technologies for the manufacturing of lighter focusing x-ray optics based on advanced material and structural concepts was clear since the beginning. In Europe ESA is pursuing the development of Silicon Pore optics (RD1) as a candidate technology for the focusing optics of next generation X-ray space observatories. This concept is based on silicon plates that are assembled into modules. These modules are integrated into segments called petals that fill the telescope aperture. The low density of $\operatorname{Si}\left(2.3 \mathrm{~g} / \mathrm{cm}^{3}\right)$ and the possibility of create high stiffness monolithic X-ray Units make this approach very attractive and very promising results have been achieved so far, even if a demonstration of imaging capabilities at a level of 5 arcsec it has not yet completely demonstrated and an effort is under way in this direction. In the mean time, the fabrication of large segmented optics based on thin shaped glass plates, obtained by hot slumping, was explored by US teams in the context of the development study of the Constellation-X mission $(\mathrm{RD} 2, \mathrm{RD} 3, \mathrm{RD} 4)$. Glass is characterized by a low density (less than $2.5 \mathrm{~g} / \mathrm{cm}^{3}$ ) and can be produced into large amount of sheets also with very low thickness (down to $0.1 \mathrm{~mm}$ or less); glass sheet can be shaped with a proper thermal cycle performed in an oven onto (or into) a mould. By means of this replica process is possible to industrially produce the large amount of mirrors segments necessary to fill the IXO telescope area. Also these developments produced very promising results, even if the focusing at a 5 arcsec HPD level based on slumped glass plates has not yet been demonstrated. Silicon pore optics and hot slumped optics are now the two main options for the realization of the IXO mirror modules. The hot slumping technique has been explored also by European teams (RD5, RD6, RD7, RD8) in the context of XEUS technological assessment studies and in the framework of the E-ELT Design Study financed by the European Community under OPTICON-FP6 for the for the manufacturing of thin optical segments for adaptive optics (RD9, RD10,RD11). ESA now considers mandatory to investigate, as a risk mitigation measure to the Silicon Pore optics, a back-up technology based on the slumped glass technique also in Europe. 
In the contest of this investigation INAF-OAB is proposing the following approach to the manufacture and integration of the grazing incidence optics for IXO. Further, to investigate all the aspects of the technology necessary for the manufacturing of the optics, it is proposing to produce a representative scaled prototype optical unit demonstrator to be tested in X-rays. This study, supported by ESA, could be developed in a timeframe of about 16 months, so to obtain an indication of the status of the proposed glass slumping technology and integration when applied to the manufacturing of X-ray optics.

\subsection{Terminology adopted for the mirrors assemblies of IXO}

To maintain a clear understanding of the whole IXO optical payload during the reading of the paper, it is important to clarify the terminology that will be used in the text. The terminology is shown in Table 2. It is defined taking into account a hierarchical principle of manufacturing and integration of the IXO Mirror Telescope which comprises the following steps:

- Fabrication of thin glass plates that have the parabolic or hyperbolic shape required for the Wolter I optical scheme and a very high surface quality

- Alignment and integration of several plates pairs in a single unit

- Alignment and integration of several single units in a suitable structure, through an intermediate level structure if necessary, in such a way to cover the entire aperture of the IXO Mirror Telescope.

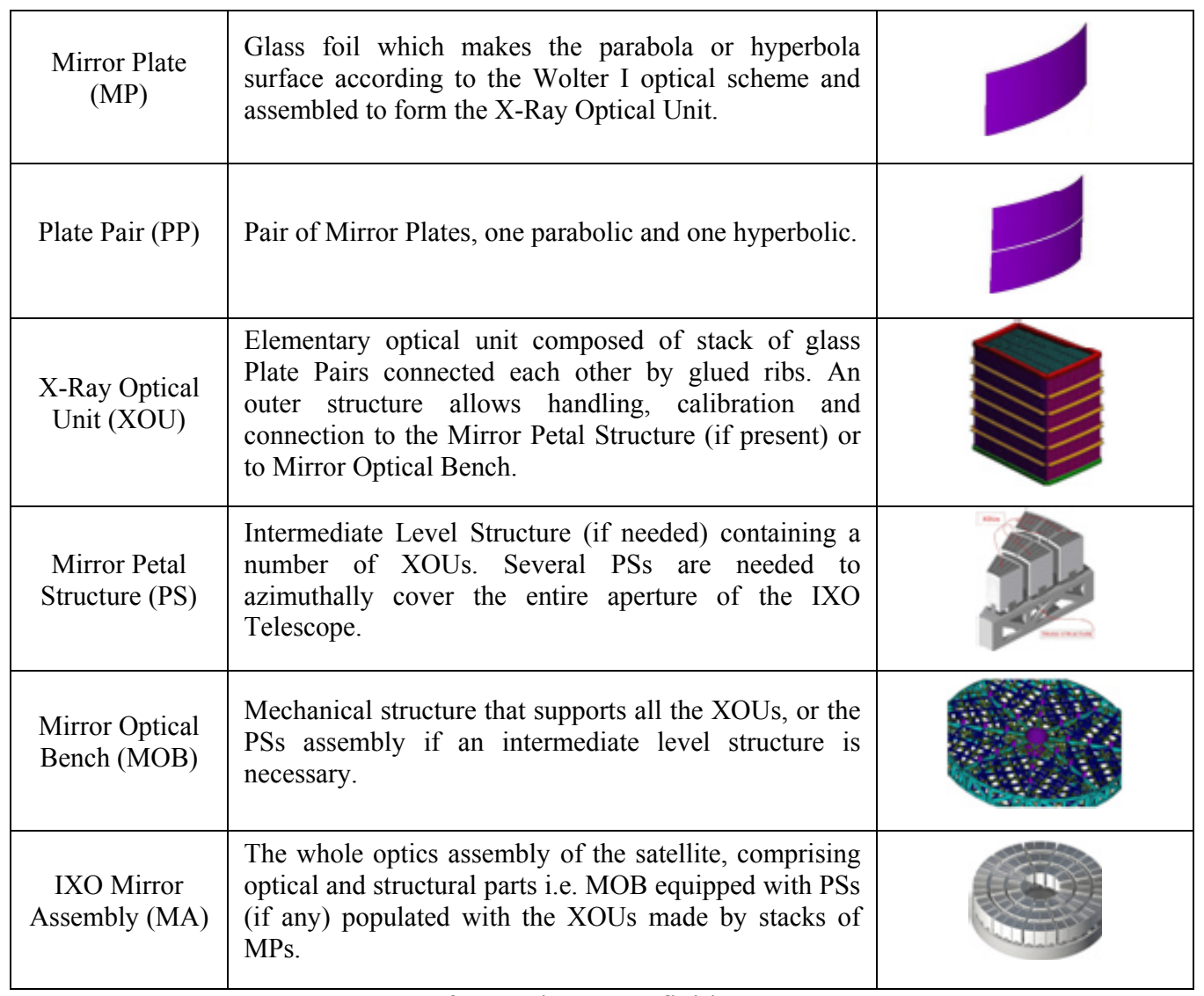

Table 2. Terminology definition 


\section{CONCEPTUAL IXO TELESCOPE DESIGN}

As previously stated, the circular shells need to be azimuthally divided into segments, named Mirror Plates, whose dimensions are compatible with the slumping technology selected to realize them. A symmetric structure of radial arms to sustain the mirrors will be the base for the partition of the telescope mirror into petals. The number of petals and the need for a further division into X-ray Optical Units (XOUs) results from a mechanical analysis of the mirror structure. The designed mirror will be the sum of several XOUs that are parts of circular coronas with dimensions optimized for reducing the obscuration area.

Considering the available geometric space and assuming a glass size of $20-30 \mathrm{~cm}$, the external corona will be composed approximately by between 32 and 36 XOUs, while the inner corona will be divided into 8-12 XOUs. An example partition of the mirror aperture into XOUs is reported in Fig. 1. This configuration is compatible with glass foils of 30 $\mathrm{cm}$ width. The obscuration of the mirror due to structures is of the order of $17 \%$, which gives an indicative value of the effective area geometric reduction in the on-axis configuration. The impact of the structure obscuration area will be analyzed with detailed ray-tracing simulations, in order to choose a design for the structure shape compatible with the requirements on the Effective Area of the mirror at different energies. The values of the Effective Area with plate pairs of $0.6 \mathrm{~m}$ (30 cm for the parabola and $30 \mathrm{~cm}$ for the hyperbola) are respectively of $5.29-5.43 \mathrm{~m}^{2} @ 1 \mathrm{keV}$ and $1.27-1.38$ $\mathrm{m}^{2} @ 6 \mathrm{keV}$ depending on the coating (400 nm of Ir or Pt). In order to fulfill the requirements related to the Effective Area at higher energy, an optimization study for the shells coating is foreseen with respect to coating material, coating thickness and multilayer coating.

Following this configuration, it has been possible to estimate the number of mirror plates and therefore the number of moulds. The number of glass Mirror Plates will be in the order of 14000, confirming the necessity of an industrial replica process for the IXO telescope mirror plates manufacturing. Those numbers are reported in Table 3 together with the estimated glass weight.

\begin{tabular}{|l|l|l|l|l|l|l|}
\hline & \multicolumn{3}{|l|}{ Shell height $600 \mathrm{~mm}$} & \multicolumn{3}{|c|}{ Shell height $400 \mathrm{~mm}$} \\
\hline Shell thickness [mm] & 0.15 & 0.30 & 0.40 & 0.15 & 0.30 & 0.40 \\
\hline Number of shells & 433 & 410 & 398 & 608 & 567 & 542 \\
\hline Glass Weight [kg] & 411 & 788 & 1030 & 390 & 742 & 954 \\
\hline
\end{tabular}

Table 3: Estimation of the number of shells and the masses of mirrors glass in case of different shell thickness and different shell longitudinal length. These numbers are obtained in case of IXO mirror segmented into four circular coronas along radial direction.

Few examples for the shape of the parabola and hyperbola respect to their cone are also reported in Fig. 2 considering the inner, medium and outer radius.
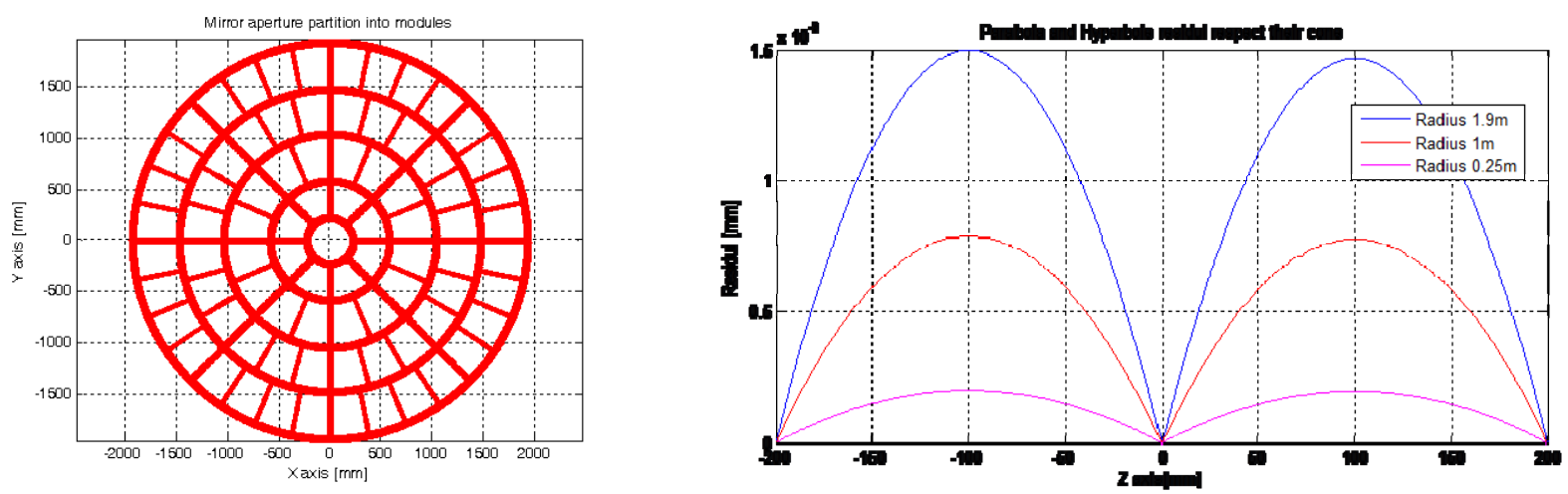

Fig 1: Partition of the mirror

Fig. 2: Parabolas and hyperbolas of three pair plates $(R=1.9 \mathrm{~m}, 1 \mathrm{~m}$ and $0.25 \mathrm{~m})$

\subsection{Preliminary conceptual mechanical design of a XOU}

From the mechanical point of view, the whole IXO mirror will be composed by an assembly of XOUs integrated on the Mirror Assembly (MA) structure. Each XOU will be composed by two stacks of glass foils which make the parabola and 
hyperbola surfaces according to the Wolter I optical scheme. The thin mirror plates (MPs) will be connected each other by glued ribs and the resulting stack, composed by MP+ ribs, will be connected to an outer structure which allows for the MPs stack handling, for the connection to the mirror assembly structures, and for the XOU calibration (Fig. 3).

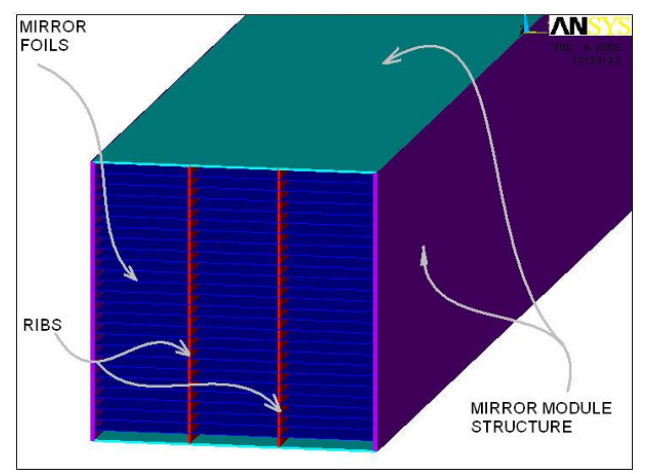

Fig. 3: XOU structure

The ribs have many functions:

1) they reduce the MP free span enabling the fulfillments of the requirements on frequencies, on strength under lift-off loads, on maximum deflection on ground during optical test;

2) they could be used also to froze eventual small corrections of the MP shape during integration phase to compensate small residual MP shape errors at the end of slumping process;

3) they could be also used for the connection of the MP stacks to the XOU outer structure.

Mechanically, a number of engineering considerations needs to be done to assess the feasibility of the proposed XOU concept. Starting from the requirements in term of maximum quasi static equivalent loads, minimum frequencies, thermal gradients in operative conditions, optical performances and strength of materials, the preliminary thermomechanical conceptual design of the XOU can be split in the following topics that will need to be investigated:

- Candidate materials trade-off for MP glass, XOU structure material, adhesive for the connections, reflecting deposit on MP. In addition to the usual mechanical properties (stiffness, density and strength) particular attention needs to be paid to the linear thermal expansion (CTE) and to the MP distortions induced by $\triangle \mathrm{CTE}$ of the coupled materials.

- Preliminary conceptual design of the main parameters as:

- Mirror plate number, size and thickness.

- Baffles length.

- Spacing between ribs and rib thickness.

$\circ \quad \mathrm{MP}$ interfaces with XOU structures. The number and the location of interfaces between MP and XOU has to be defined.

- Possible structural schemes of XOU structure in order to fulfill structural and optical requirement.

- Preliminary design of the XOU structure.

All these investigations can be carried on by proper finite element models or FEA., whose level and complexity can be gradually increased according to the needs.

Concerning to the strength of the glass material it has to be noted that the strength of these materials is not an intrinsic and fixed characteristics of the material itself, being strictly affected by many variables as for instance the defects present in the glass at the surface and the kind of load (static, dynamic). So the glass strength varies with the particular manufacturing process, with the load, with glass zones (for instance it could be lower at foil edges due to the higher probability of defects). Furthermore the glass strength show a significant statistical dispersion and usually it is characterized, in statistical terms, by Weibull parameters which in principle should be computed, by a long test series, for each particular application (i.e. manufacturing process). During the proposed study a limited number of strength tests under static loads on simple glass plates, obtained with the same slumping process to be used for the XOU, could be realized to check if their results are compliant with the strength criteria assumed in the design process. 


\section{SLUMPING OF SINGLE IXO MIRROR PLATES}

\subsection{Slumping technologies state of the art}

The slumping technology foresees the realization of glass MPs by means of a thermal treatment of a glass sheet lying over a mould that has the desired shape. The advantage of this technology is that it is a replication process i.e. one mould can be used several times to realize a number of mirrors starting from commercially available glass materials. At temperatures between the annealing point and the softening point, the viscosity of the glass is such that it slumps into (or onto) a given mould. Above the softening point the glass becomes too soft (essentially melts) and looses its original surface properties. Table 4 summarizes some characteristics of materials that can be used to manufacture moulds.

Property
\begin{tabular}{|l|l|l|l|}
\hline Elastic Modulus $(\mathrm{GPa})$ & 90 & 476 & Sused Silica \\
\hline Knoop Hardness $(\mathrm{HK} 0.1 / 20)$ & 1900 & 3000 & 580 \\
\hline CTE $\left(10^{-6} / \mathrm{K}\right)\left(\mathrm{RT}\right.$ to $\left.1000^{\circ} \mathrm{C}\right)$ & 8.2 & 4.0 & 0.5 \\
\hline CTE homogeneity $\left(10^{-6} / \mathrm{K}\right)$ & Good & Good $(\Delta=0.01)$ & Good $(\Delta=0.01)$ \\
\hline Thermal cond. $(\mathrm{W} / \mathrm{mK})$ & 24 & 102 & 1.51 \\
\hline Glass adhesion & YES & YES & YES \\
\hline Microroughness & $10-20 \AA$ & $<5 \AA$ & $<5 \AA$ \\
\hline Polishability and figuring & Slow & Very slow & Fast \\
\hline Machinability & In green body & In green body & Very good \\
\hline Stability after cycles & Very good & Very good & Very good \\
\hline
\end{tabular}

Tab. 4: Characteristics of some possible materials to manufacture moulds

The slumping is done at temperatures between the annealing point and the softening point in order for the glass to slump without loosing its original good surface properties. The thermal cycle may be different, but it always includes a number of ramps (increasing and decreasing in temperature) and plateaus at which the temperature is maintained constant. The heating can be relatively fast up to the required slumping temperature: then an holding time ensure that no temperature gradients are present and the glass has time to slump against the mould. The cooling down will be done slowly to give the glass the possibility to reach the annealing point without internal stresses, since below this point the glass is no longer able to relax them. Table 5 reports different glass types available on the market, their principal properties and available dimensions.

\begin{tabular}{|l|l|l|l|l|l|l|l|l|}
\hline Parameter & AF32 & AF37 & AF45 & D263T & 0211 & 1737F & Eagle2000 & Bonfloat \\
\hline Density $\left[\mathrm{g} / \mathrm{cm}^{3}\right]$ & 2.43 & 2.48 & 2.72 & 2.51 & 2.53 & 2.54 & 2.37 & 2.2 \\
\hline YoungMod. $\left[\mathrm{kN} / \mathrm{mm}^{2}\right]$ & 74.8 & 78 & 66 & 72.9 & 75.9 & 71.4 & 70.9 & 64 \\
\hline CTE $\left[10^{-6} / \mathrm{K}\right]$ & 3.2 & 3.77 & 4.5 & 7.2 & 7.38 & 4.2 & 3.6 & 3.25 \\
\hline Strain point $\left[{ }^{\circ} \mathrm{C}\right]$ & 686 & 684 & 627 & 529 & 508 & 666 & 669 & 518 \\
\hline $\begin{array}{l}\text { GlassTransition } \\
\text { Log } \mu^{*}=14.5\left[{ }^{\circ} \mathrm{C}\right]\end{array}$ & 715 & 711 & 662 & 557 & n.a. & n.a. & n.a. & 525 \\
\hline $\begin{array}{l}\text { Annealing point } \\
\text { Log } \mu^{*}=13.0\left[{ }^{\circ} \mathrm{C}\right]\end{array}$ & 728 & 722 & 663 & 557 & 550 & 722 & 722 & 560 \\
\hline $\begin{array}{l}\text { Softening point } \\
\text { Log } \mu^{*}=7.6\left[{ }^{\circ} \mathrm{C}\right]\end{array}$ & 969 & 942 & 883 & 736 & 720 & 975 & 985 & 820 \\
\hline Thickness $[\mathrm{mm}]$ & $0.1-1.1$ & 0.7 & $0.05-1.1$ & $0.03-1.1$ & $0.05-0.5$ & $0.5-1.1$ & $0.5-0.7$ & $\geq 0.7$ \\
\hline Thick. const. $[\mu \mathrm{m}]$ & \pm 5 & \pm 50 & $\pm 10-50$ & $\pm 8-50$ & n.a. & n.a. & \pm 20 & \pm 70 \\
\hline Linear Dims. $[\mathrm{mm}]$ & $440 \times 360$ & $2160 \times 2400$ & $440 \mathrm{x} 360$ & $440 \mathrm{x} 360$ & $406 \times 432$ & $355 \mathrm{x} 457$ & $1100 \times 1250$ & Up to 3000 \\
\hline
\end{tabular}

$* \mu=$ viscosity of the glass - AF32, AF37, AF45, D263T, Borofloat are glasses manufactured by Schott; 0211, 1737F and Eagle2000 are manufactured by Corning

Tab. 5: Characteristics of thin glass sheets that is possible to use to realize MPs

These are industrially manufactured borosilicate glasses that come from the display industry with thickness smaller than $1 \mathrm{~mm}$. Only thin thickness glasses can be used due to mass limitation required by the mission launch. Borofloat is reported for comparison, it has been largely used for the development of the slumping technology, but it is not applicable to the IXO optics because it is too thick. Among these glasses, D263T has been largely characterized for space applications (RD12) while AF45 is under space characterization by American groups. For what concern the mould material, the Silicon Carbide ( $\mathrm{SiC}$ ) is very interesting for its thermo-mechanical characteristics because it has a thermal 
conductivity similar to that of a metal, a characteristic that in principle permits to speed the slumping process because eventual thermal gradients on the mould (and glass) are quickly dispersed. As drawback the SiC is very expensive, difficult to machine and tends to stick to the glass at the temperature used during the slumping. To overcome this problem an anti-sticking layer is necessary. A possible alternative for the mould material can be found in the Fused Silica that is a well known material for lenses, easily grinded and figured and able to resist to very high temperatures. It, as the $\mathrm{SiC}$, tend to stick to the glass necessitating an anti-sticking layer too. Fused Silica has a low thermal conductivity which requires low cooling rates to avoid temperature gradients in the mould.

During this study, two slumping approaches will be considered (Fig. 4):

- Direct Slumping: the optical surface of the MP is the side of the glass plate that comes in contact with the mould during the slumping. For the purposes of this study it will be based on the use of convex moulds. A pressure is actively applied to force the glass to come in contact with the mould.

- Indirect Slumping: the optical surface of the MP is the non-contact side of the glass. For the purposes of this study it will be based on the use of concave moulds. The glass slump on the mould using its own weight (by gravity)

\section{indirect}

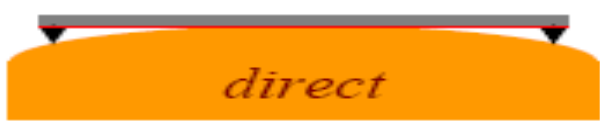

Fig. 4: Indirect and Direct slumping concepts

It should be noted that the Direct Slumping approach studied so far in Europe by INAF-OAB is different from the technique currently investigated in USA at NASA/Goddard Space Flight Centre and Centre for Astrophysics. The process investigated by the American Institutes foresees the use of a convex mould onto which the glass slumps only under its own weight without the application of additional forces. This requires to trim (cut) the wings of the segment after the slumping because the weight of the glass alone is not enough to bring it in full contact with the mould in that outer parts. Instead the Direct Slumping approach adopted in the present study employs an active pressure system to force the glass to full contact with the mould also on the edge areas of the glass. The technique was developed in INAFOAB during a previous study financed by Opticon FP6 in the contest of the slumping of optical surfaces for adaptive optics giving very good results in view of the application for X-ray optics. Further, the American approach foresees the deposition of the reflecting coating after the slumping, a step that has the potential to add stresses to the glass and change its overall optical shape. Instead (as later described) the proposed direct approach uses Pt or Ir films as anti-sticking layer AND X-ray reflecting surface in which stresses are relaxed after the thermal cycle.

\subsection{Direct Slumping current results}

The Direct Slumping process is shown in Fig. 5.

1. The convex mould is grinded and figured with the required shape and very low microroughness (less than 5 A rms)

2. The glass sheet is coated with an anti-sticking layer like Pt or Ir. At the end of the process this will be the $\mathrm{X}$-ray reflective layer.

3. The glass sheet is placed above the mould in a muffle from where the air is evacuated.

4. The thermal cycle is started and at temperatures between the annealing point and the softening point, a uniform pressure is applied on the glass in order to push it against the mould and copy its shape. The pressure is applied by means of a proprietary process developed by INAF-OAB.

5. After the cooling of the oven the slumped glass sheet is released with the reflective layer still on it.

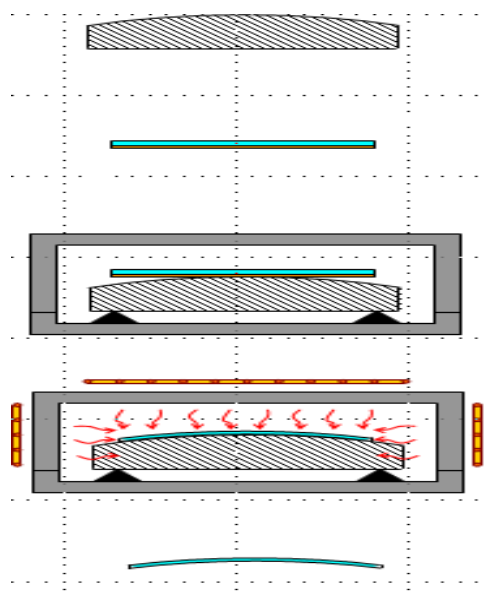

Fig. 5 Direct slumping process 
The process takes place in a stainless steel muffle with vacuum in which the glass and the mould are protected from the dust of the oven. The cleaning preparation of the muffle and its content is done in a clean room environment to minimize the risk of dust contamination. In the vacuum muffle convection is minimized permitting a uniform temperature distribution and small thermal gradients. To avoid the possibility of sticking of the glass on the mould, a separation layer may be needed depending from the mould material. For chemical reasons this problem arises in particular for moulds made in SiC or Fused Silica. The use of Pt or Ir coating on the glass sheet before the slumping not only prevents this problem but, after the separation, it acts as the final X-ray reflecting surface of the mirror. Moreover the stresses that are normally present in a reflecting film after the deposition are partially relaxed due to the thermal cycle that also increases the layer adherence to the glass. This situation is similar to the case of the Au layer in the Ni electroforming replica process used for example for the production of the monolithic XMM shells, having the simultaneous functions of release agent from the mandrel and X-ray reflecting coating.

This slumping approach has given very promising results and has been developed making use of K20 mould material (a special crystallization brand of Zerodur) and Borofloat glass. Currently, tests have been performed on spherical shape convex moulds with radius of curvature between 5 and $10 \mathrm{~m}$. Disks of Borofloat having diameters of $130 \mathrm{~mm}$ and 500 $\mathrm{mm}$ respectively and thicknesses in the range of 1,7-2 $\mathrm{mm}$ were used. It has demonstrated the capability to copy not only the low frequency profile of the mould geometry, but also the surface topography at high spatial frequencies.
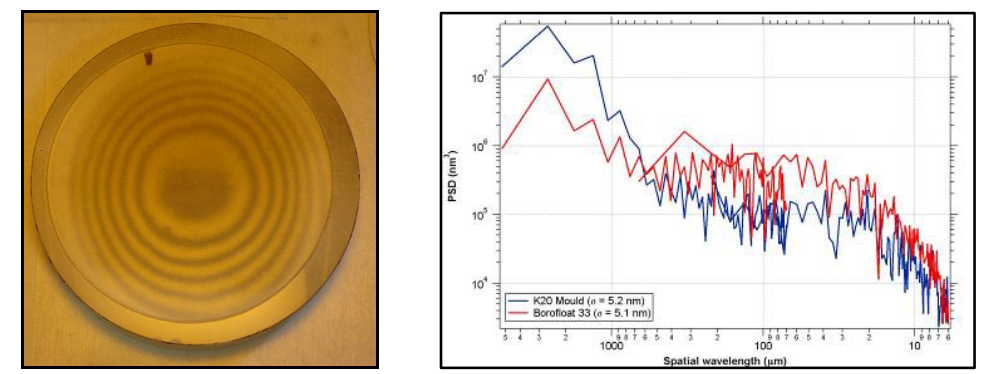

Fig. 6: Interference fringes between a spherical mould and the glass after a slumping. - PSD of the slumped glass

In Fig. 6 is visible a slumped glass disk of $130 \mathrm{~mm}$ diameter and $2 \mathrm{~mm}$ thickness placed onto the mould used for the slumping. Under Sodium light a number of circular fringes are visible. The circularity of the fringes indicates that the two surfaces (mould and glass) differ only in the radius of curvature but the sphere geometry was copied very well. This effect was expected due to the CTE difference between mould and glass. Also higher spatial frequencies were copied as it is visible in the Power Spectral Density (right panel).

Tests made on the K20 mould have produced repetitive performances of shape in the slumped glass disks producing thin glass shells with residual errors respect to the interferometer spherical null of 1/2 - 1/3 lambda rms (@633 nm). The intrinsic error of the mould shape was similar, indicating that the process performances were limited by the mould quality. By using a larger mould in K20 we have been able to copy its $250 \mathrm{~mm}$ central part area obtaining again a surface accuracy of the slumped shell of $1 / 2$ lambda. These results are very encouraging because again the copy capability was limited by the mould accuracy, not by the technique. A pseudo cylindrical surface like that of a Wolter I optic should be easier to copy than a sphere (the bending on a sphere is of the same magnitude on both axis) giving confidence that using good shape quality moulds will produce high quality thin MPs.

\subsection{Indirect slumping current results}

The Indirect approach has been investigated in the past few years by MPE and INAF-OAB with the support of Schott Glass Company. In Fig. 7 is visible the muffle used during these slumping tests with a K20 cylindrical mould and a glass plate on it. A representative slumping result is shown with an accuracy on the slumped plate of 7 microns of residual shape error with respect to the cylinder. The indirect slumping technology is currently being further investigated at MPE and recent results are indicated in (RD5, RD6, RD7). It uses the gravity to slump the glass into a concave mould. During the study of this technique it has been shown that the back side of the glass comes completely in contact with the mould surface and thus reproduces well its geometric figure. The main advantage of the Indirect Slumping is that the mould (concave) needs only a good geometry, not a good microroughness, because the reflective surface of the glass plate is the upper one, not in contact with the mould surface. The smaller the structures on the mould are, the more they are damped through the thickness of the glass. Also, the intrinsic good microroughness of the glass surface is preserved. On the other hand a major drawback is the fact that the glass sheet has intrinsic thickness variations (due to the glass manufacturing 
process) that cause corresponding figure errors on the upper surface when the glass back side is in full contact with the mould. These variations degrade the optical figure of the segment and have a range of a few microns (for D263T). To avoid this effect it is necessary to start with a glass sheet having very uniform thickness (less than $0.5-1$ micron on large scales). Therefore the glass needs to be grinded or polished at least on one side before its use for the slumping.
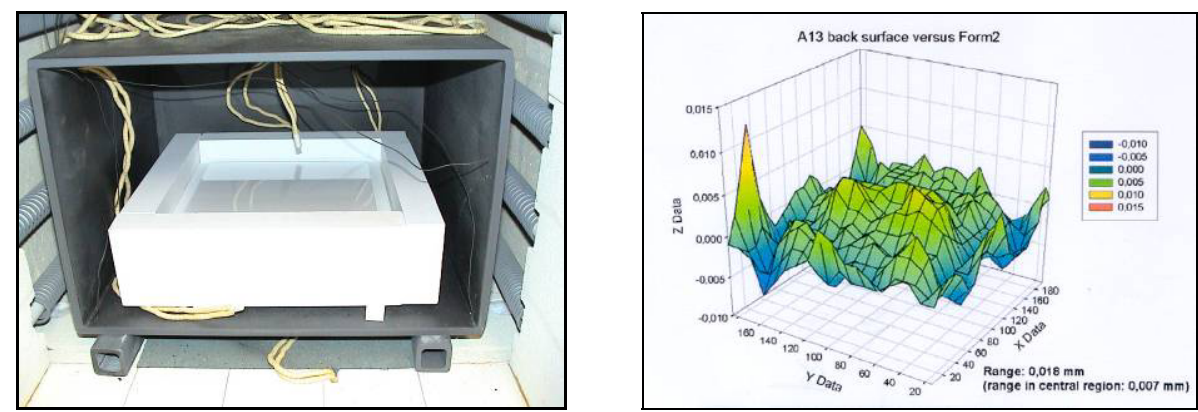

Fig. 7: K20 cylindrical mould and slumping result

In the study performed so far, industrially manufactured borosilicate glasses were used which fulfill the conditions of low micro-roughness and thickness: in particular the display glass D263T and the glasses AF32 or AF45 are available with thickness from about 0.03 to $1.1 \mathrm{~mm}$ and having thickness variations up to 6 microns. Both glass types are manufactured by Schott.

The Indirect Slumping process is done as follow:

1. The concave mould is grinded and figured with the required shape.

2. The glass segment is coated with an anti-sticking layer like Pt or Ir film. At the end of the process this will be removed by chemical action.

3. The glass sheet is placed above the concave mould directly in the oven since the dust problem is less critical for the indirect approach.

4. The thermal cycle is started and at temperatures between the annealing point and the softening point the glass slumps under its own weight into the mould copying its shape.

5. After the cooling of the oven the MP is released from the mould and the antisticking coating is removed, if present.

6. The MP is coated with the $X$-ray reflective layer.

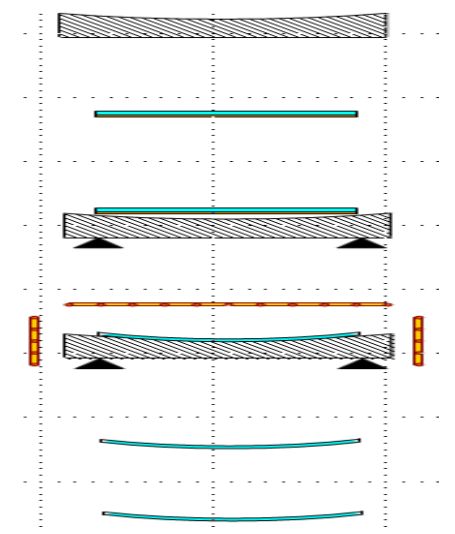

Fig. 8: Indirect slumping process

In Fig. 9 is visible an example of slumping. A green aluminum oxide ceramic mould is in the oven with a slumped segment placed above the mould after the slumping. The fringes at the edges of the glass indicate that there is a small gap between glass and mould (after having been in full contact during the slumping) which is due to the slightly higher thermal expansion coefficient of the glass. There is a horizontal line located in the middle of the segment which marks the intersection line between the parabolic and the hyperbolic part of the mould.
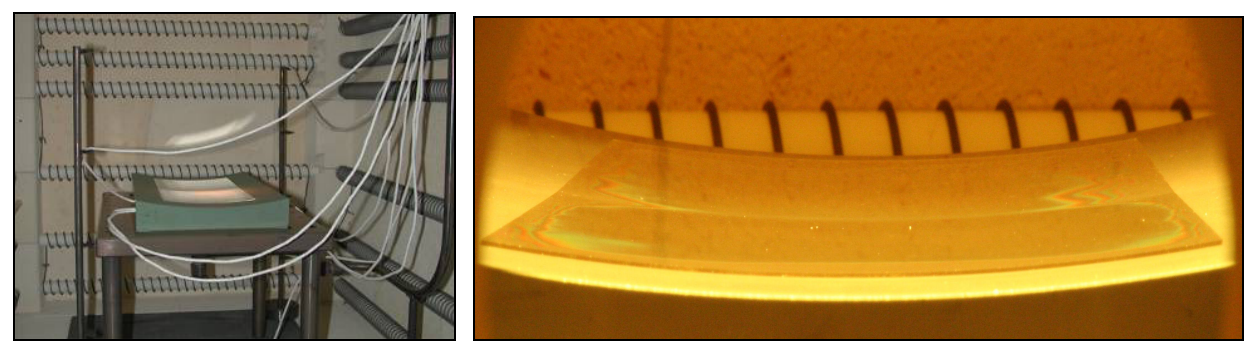

Fig. 9: Ceramic mould and Indirect slumped glass segment after cooling 


\subsection{Comparison of different glass slumping techniques and tradeoff}

Direct and Indirect slumping techniques characteristics are here summarized:

\section{DIRECT SLUMPING}

$\circ \quad$ Constant thickness of glass not required

- The use of pressure guarantee full copy of the whole plate area

- Dual use of Pt or Iridium: separation layer AND X-ray reflective surface (lower stresses)

$\circ$ Very low microroughness of the mould surface is requested

\section{INDIRECT SLUMPING}

$\circ \quad$ No need of super polished mould surfaces

- Full contact of the glass foil with the mould only by gravity is feasible

- Very high thickness constancy of the glass required

- Reflecting layer to be deposited after the slumping (possible stresses)

During this study INAF-OAB will use both slumping methods (direct and indirect) for the slumping of test segments and will compare them to find the best technique to be used for the slumping. This tradeoff phase will select the technique to be used for the production of the MPs to be integrated into the XOU scaled prototype.

For the comparison the slumped segments will be characterized in terms of:

- $\quad$ Glass slumped plate geometry, compared with the geometry of the mould.

- $\quad X$-ray microroughness measurements of the slumped glass plates.

The copy capability of the two slumping techniques can be demonstrated using cylindrical moulds that are much more easy to be produced than the Wolter I profiles. A possible setup for the geometry measurement of a slumped plate make use of an interferometer with a Computer Generated Hologram (CGH) as shown in Fig. 10. During the shape characterization the plates will be sustained by an astatic support that will remove the shape deformations due to the gravity, leaving only the intrinsic shape of the plate during the measurement (RD8).

The microroughness measurements of the slumped segments can be be done using Atomic Force Microscope, Interferometric profilometers and X-ray scattering instrumentation available at INAF-OAB.

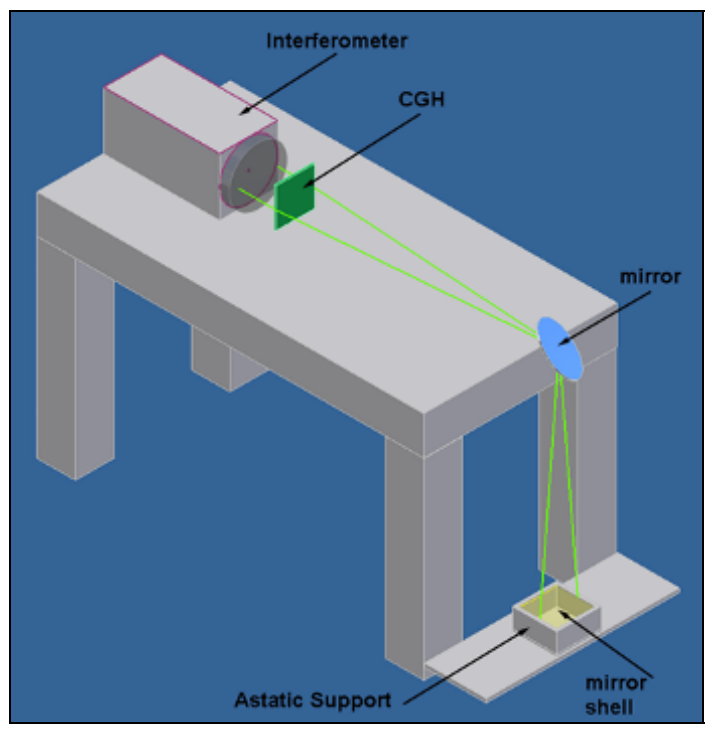

Fig. 10: Setup for interferometric tests of slumped MPs with astatic support. 


\section{ALIGNMENT AND INTEGRATION OF MIRROR PAIRS INTO THE XOU}

Whichever technique is used for the slumping of mirror plates, they have to be aligned and assembled into an XOU. The proposed conceptual mounting scheme of the MPs into the XOU is shown in Fig. 11. It is based on a direct optical feedback at the focal plane that allows the monitoring of optical performances during the integration phase.

- A pair of MPs will be placed above the integration tools (IT) using reference markers for alignment.

- Vacuum suction will be applied to fix the plates against the tools and reinforcing ribs will be glued to the back of the plate.

- Collimated light and grazing incidence illumination of the MPs will be used to obtain a focal spot through grooves cut longitudinally into the suction tools.

- Actuators to control tilt and position between the two suction plates will be used to adjust the focus and correct the alignment between the MPs.

- Once the PP is in position, the ribs will be glued to the backplane. Then the vacuum suction will be released.

- The process will be repeated for all the PPs to be mounted in the XOU, always using as reference the focal spot of the first PP mounted.

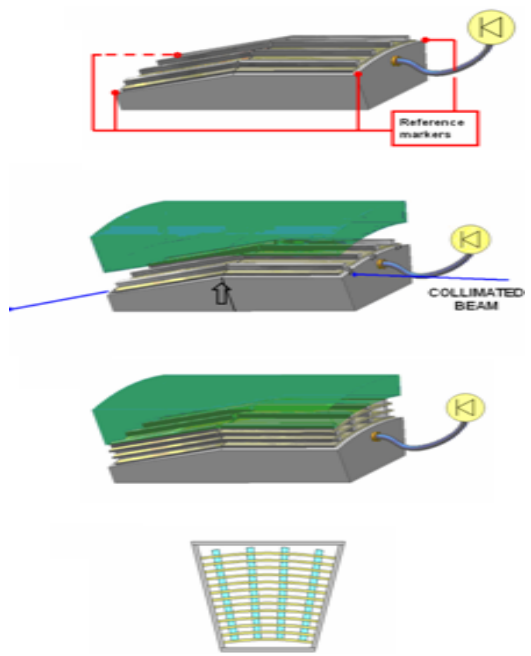

Fig. 11: Conceptual mounting scheme of mirror plates pair into the XOU module

We plan to follow the conceptual scheme just described using the following technical solution.

Mirror Plates alignment on the Integration Tools (IT). As a first step each slumped MPs will be placed on its IT. The latter is a mandrel made of "porous graphite" (Fig. 12) having the same geometrical shape of the slumping mandrel used to make the MP, in order to apply a uniform suction force. The porous graphite material is cheap, easily machined and figured.
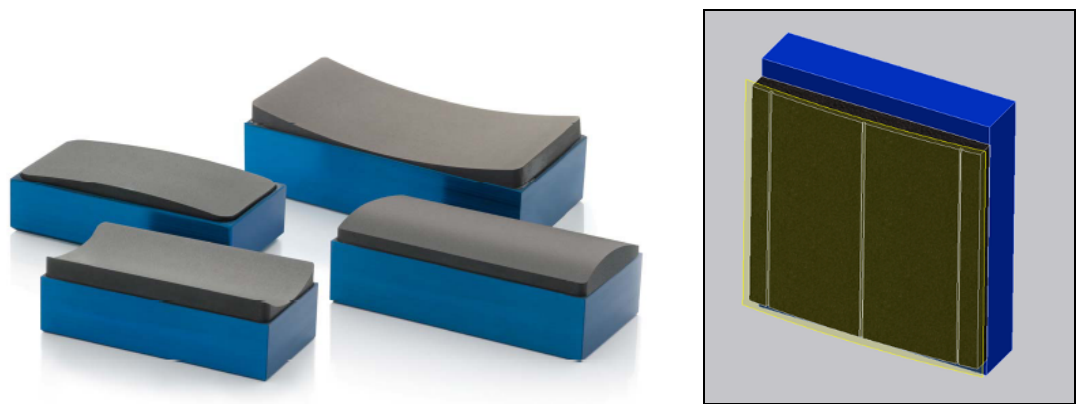

Fig. 12: Porous graphite (New Way Airbearings) and view of an IT with three grooves and a MP placed on it.

The shape of the MP on the IT will be essentially equal to the intrinsic MP shape. The alignment of each MP on its IT will be made under a microscope by means of markers. Markers will be obtained on the plates by making small spots on the slumping mould, so that they will be copied on the plates. The markers will be done on the mould and the graphite tool by means of a laser scribe with a suitable shape like for example a bull eye with a final centre point having dimension of about 20 microns. By making a centroid estimation of this central point it will be possible to reach an alignment accuracy between MP and IT of few microns so to avoid to introduce relevant distortions in the MP. Once each plate is placed on its IT, the vacuum plant is activated and the plate is sucked onto its tool fixing it in place. When the MPs are ready and under vacuum suction, a number of glass reinforcing ribs (determined by the results of the thermo-structural analysis) will be longitudinally glued on them in predetermined positions using a specialized tool. Then, the ITs (and the plates with the ribs) will be installed on the Integration Frame.

Integration Tools alignment in the Integration Frame (IF). The IF is an Aluminum structure that allows placing the ITs in a given relative position and attitude. Initially the parabola IT is placed in position on the IF: by means of 
mechanical references it is planned to achieve a $0.05 \mathrm{~mm}$ and 1 arcmin positioning accuracy. Then the hyperbola IT is placed on the integration frame in a similar manner. The IF (Fig. 13) embeds three controlled degrees of freedom to allow active alignment of the hyperbola with respect to the parabola. In particular, assuming the Coordinate System of Fig. 15 we control the Rx, Ry and Rz rotations. This is done by means of three rotary stages that uses pre-loaded flexure pivot points and submicron linear actuators placed at a given arm distance to the relevant pivot axis. Further, commercial hexapods are capable to position a payload under $50 \mathrm{Kg}$ mass with a submicron and arcsec accuracy. Such numbers together with the features of the three rotation degrees of freedom embedded under the hyperbola IT are well enough to achieve the demanded positioning and attitude accuracy of the MPs into the XOU. Note that it is proposed to make the whole installation process having the plates facing down. This configuration ensure a passive safety in case of failure of the porous graphite suction system.
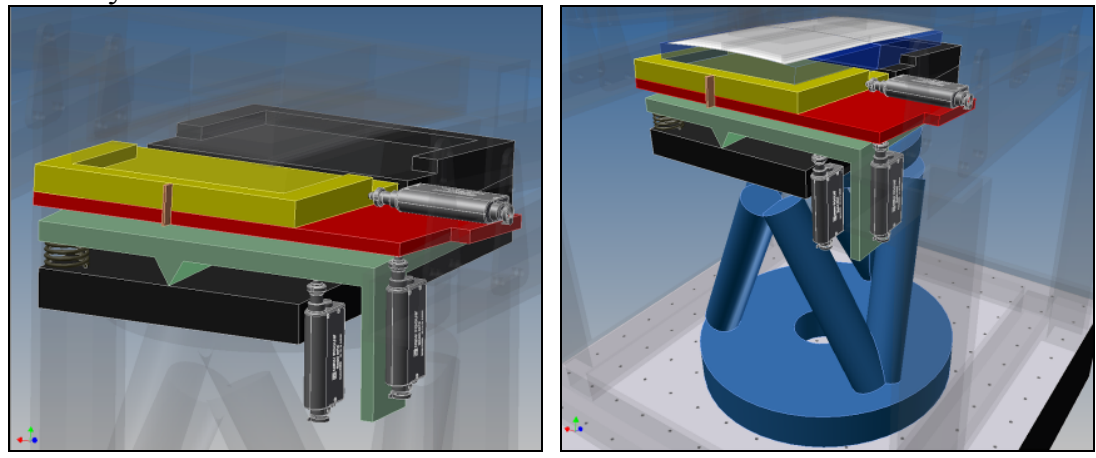

Fig. 13: The IF seen without the two ITs and mounted onto hexapod

MPs final alignment into the XOU with focal plane metrology. The IF will be placed below a glass Backplane on which the PPs will be integrated. This Backplane (Fig. 14) will be placed into the beam of an interferometer able to illuminate also all the PPs. The backplane will have a flat surface optically figured, facing toward the interferometer: in this way, it will be possible to put this face perfectly perpendicular to the interferometer beam (zero fringes). This essentially is equivalent to be able to keep fixed and controlled the angle between the PPs (that will be attached to the Backplane) and the interferometer beam. This capability is necessary to guarantee the repeatability and stability of the focal spot position. The integration suction tools will have three "channels" or "grooves" aligned with the optical axis. Three pencil beams from the interferometer will pass through them, reflected on the MPs at grazing incidence and then focused on the XOU focal plane.
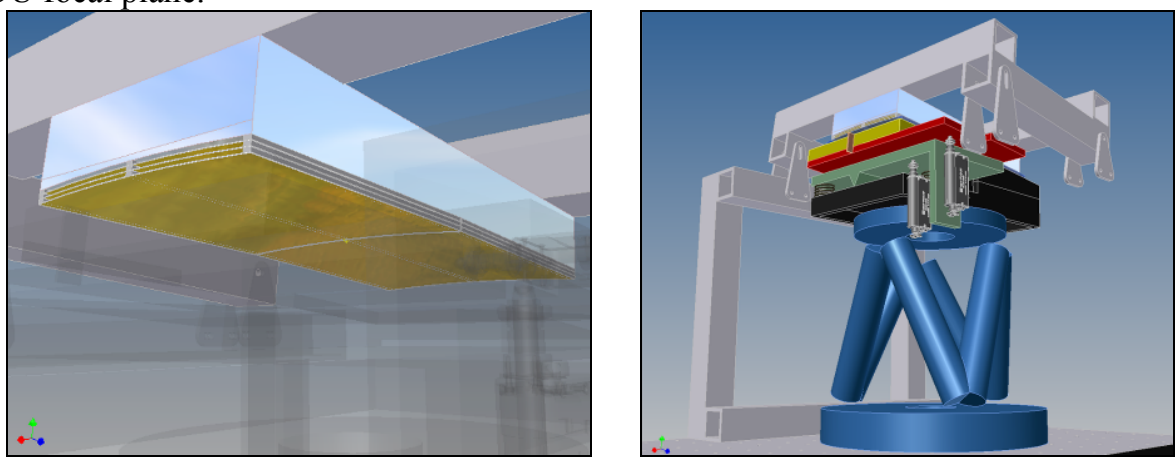

Fig. 14: Backplane and general assembly of the IMA. Hexapod is visible together with the three axis IF.

Due to the diffraction, at the focal plane every pencil beam will generate a focal spot having a diameter of about $16 \mathrm{~mm}$. The centroids of the three pencil beams obtained alternatively obscuring two of the three grooves should be focused in the same position in the case of a perfect PP geometry. The baricenter that will be used as final position for the focal spot of the PP under evaluation will be the mean position of the three centroids.

Initially the relative position between the two MPs on the IF is obtained within the tolerances by mechanical means. It is checked measuring the distances between the edges of the two plates nearest to the intersection plan with zoom cameras located in suitable positions.

The PPs alignment will be an iterative procedure using the focal plane metrology (see Fig. 15 for better understanding): 
- The IF with the ITs is lifted and aligned with respect to the Backplane by means of a 6-degrees of freedom parallel mechanism, hexapod. The parabola MP will be placed leaving a small gap (controlled with cameras) between its ribs and the Backplane surface.

- The hyperbola MP is rotated around the Y axis by means of the actuator of the IF. With the rotation along $\mathrm{Y}$ the light from the interferometer will reach the focal spot going onto a CCD sensor (about 30x30 mm) placed in the mechanically predetermined nominal position on the focal plane. This rotation will move the focal spot along the $\mathrm{X}$ axis of the CCD. The spot centroid will be placed in the centre of this axis.

- Rotating the hyperbola plate along its $Z$ axis the focal spot will be moved along the $y$ axis of the CCD sensor. With the hyperbola plate correctly aligned respect to the parabola plate along the $\mathrm{Z}$ axis, the spot centroid will be placed in the centre of the CCD Y axis.

- Rotating the hyperbola along the $\mathrm{X}$ axis of the IF the focal spot will elongate symmetrically on the axis $\mathrm{X}$ of the CCD. It will be necessary to find an angular position in which this elongation has the minimum extension.

After these four steps the PP will be in the ideal position (relative position between parabola and hyperbola and also in focal spot position) having the focal spot centroid in the centre of the CCD (assuming the CCD is perfectly centered on the focal plane).

Once the first PP with its ribs is aligned into the XOU, it is glued to the Backplane. The process is then repeated for the next PPs. This procedure creates a stack of PPs whose focus spots are over-imposed onto the first one that is used as reference. Note that the proposed method allows to align each new PP with respect to the first one integrated into the XOU, thus the final integration accuracy will not depend on the number of shells mounted into the XOU itself.

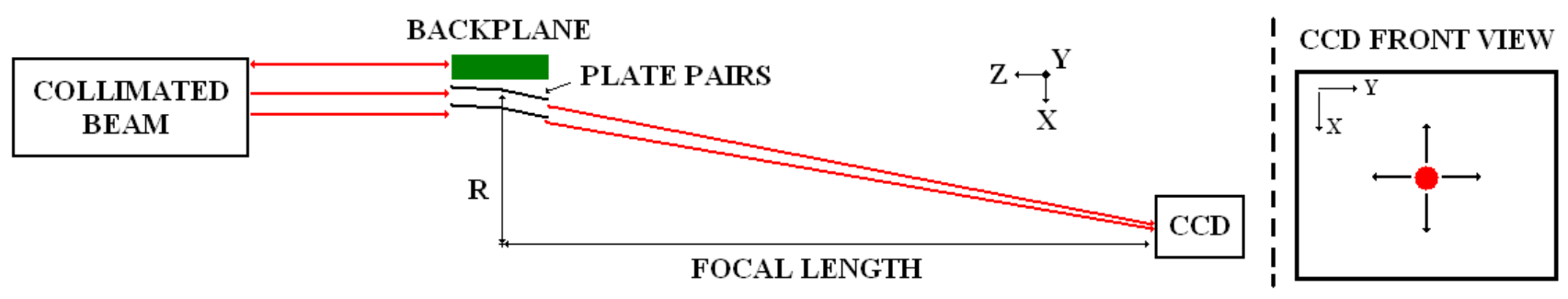

Fig. 15: Optical scheme for focal plane integration.

\section{XOU SCALED PROTOTYPE FOR THE IXO TELESCOPE}

The XOU scaled prototype that is here proposed to build will consist of one stack of three adjacent Wolter I glass PPs integrated into a mechanical structure containing a total of 20 PPs. The three PPs will be the test ones, slumped and integrated using the concepts till here described. The other 17 PPs will be also made in glass but "dummy" and their purpose is to permit to create a mechanical structure somewhat representative of a real XOU. The specs proposed for the XOU mirror plates are: Dimensions of 200x200 mm; Thickness 0.3-0.4 mm; Radius of curvature: $1 \mathrm{~m}$; Glass type selected from: D263T, AF32, AF 45 (from Schott).

Assuming that the IXO_MA will have a radial partition into four main circular coronas, then the second segment of circular coronas is a good compromise for being representative of the mirror technology development because of its intermediate size. In Table 6 are reported the values of the characteristic radii of the shells: at entry pupil, at intersection plane and at exit pupil.

\begin{tabular}{|c|c|c|c|c|}
\hline $\begin{array}{l}\text { Entry pupil } \\
{[\mathrm{mm}]}\end{array}$ & $\begin{array}{l}\text { Intersection plane } \\
{[\mathrm{mm}]}\end{array}$ & Exit pupil [mm] & $\begin{array}{l}\text { Angle } \\
{[\mathrm{rad}]}\end{array}$ & Shell \# in XOU \\
\hline 1006.19 & 1003.69 & 996.16 & 0.012536 & 156 \\
\hline 1009.30 & 1006.79 & 999.00 & 0.01257 & 157 \\
\hline 1012.42 & 1009.90 & 1002.33 & 0.012613 & 158 \\
\hline
\end{tabular}

Tab. 6: Radius of the PPs that will compose the XOU scaled prototype 
Assuming a Wolter I geometry, the system has a perfect image at the focal plane when perfectly integrated and with ideal surface plates. Considering the same system placed at a finite distance (130 $\mathrm{m}$ as at the Panter facility) and rotated of -30 ' around axis $\mathrm{Y}$, the system will have a focus at $23654.5 \mathrm{~mm}$.

Inside the XOU prototype, as in each XOU, the PPs surfaces are separated by a minimum gap at the level of the intersection plane in order to allow the independent slumping and integration of the two MP. Since the two corresponding surfaces are separated, it will be necessary to study the impact of the alignment errors (misalignment and tilts) of the optical surfaces in terms of imaging quality and angular resolution. Ray tracing simulations will be used for the alignment requirement definition that will be part of the error budget tree. Constrains on the integration errors of each MP segment, both for a PP system and for the whole XOU, will be the base for the integration and alignment procedure scheme. A preliminary investigation by ray tracing analyzing the behavior of the focal plane spot and considering that the total amount of the integration error is 2", has given a first estimate of the integration tolerances of the MPs. These are given in Table 7.

\begin{tabular}{|l|l|l|}
\hline & Parabola MP - Max Integration tolerances & Hyperbole MP - Max Integration tolerances \\
\hline Rot. around X & $\pm 3^{\prime}$ & $\pm 3{ }^{\prime}$ \\
\hline Rot. around Y & $\pm 0.2^{\prime \prime}$ & $\pm 0.2^{\prime \prime}$ \\
\hline Rot. around Z & $\pm 16^{\prime \prime}$ & $\pm 16^{\prime \prime}$ \\
\hline Displ. along X & $\pm 80 \mu \mathrm{m}$ & $\pm 30 \mu \mathrm{m}$ \\
\hline Displ. along Y & $\pm 80 \mu \mathrm{m}$ & $\pm 80 \mu \mathrm{m}$ \\
\hline Displ. along Z & $\pm 3 \mathrm{~mm}$ & $\pm 700 \mu \mathrm{m}$ \\
\hline
\end{tabular}

Tab. 7 Maximum tolerances for integration.

Metrological characterizations of the produced MPs, will pay particular attention to the shape accuracy and microroughness of the glasses before/after slumping. The mirror integration in the supporting structure is not expected to introduce surface smoothness degradation, while it might significantly affect the mirror profile. For this reason, and due the problematic access to the optical surfaces with the metrological instrumentation after integration, most of the characterization for the XOU demonstrator should be demanded to the X-ray full illumination measurements to be performed at the Panter facility. Metrological analysis at INAF-OAB will comprise:

1) Mirror figure mapping along with characterization in visible light (@633 nm), using the ZYGO® interferometer available at INAF-OAB. The surface mapping will be executed before and after the slumping to assess the compliance of the profile imparted to the glass surface with the specification.

2) Roughness morphology inspection by means of the phase-contrast microscope (mod. Nomarsky) with a vertical resolution of a few nanometres.

3) Microroughness measurements along with interferometry in visible light (white spectrum) using the WYKO ${ }^{\circledR}$ microscope able to detect the surface roughness profiles in the low middle frequency range (a few $\mathrm{mm}-10 \mu \mathrm{m}$ ), with a resolution of few Angström.

4) Microroughness measurements in the high frequency regime $(50 \mu \mathrm{m}-10 \mathrm{~nm})$ using an Atomic Force Microscope able to perform surface mapping (100 $\mu \mathrm{m}$ max. size) with a lateral resolution of a few $\mathrm{nm}$ and a vertical resolution better than $0.5 \AA$.

5) X-ray characterizations in pencil beam ( $50 \mu \mathrm{m}$ beam width) setup, either in monochromatic $(8.05 \mathrm{keV})$ or energydispersive $(5-50 \mathrm{keV})$ mode. Measurements of X-ray reflectivity will allow to measure directly the optical performances of the mirror glass, whereas X-ray scattering measurements $(8.05 \mathrm{keV}$ only) will return an independent measurement of the roughness power spectrum with high statistical significance, to be compared with the results of the methodologies mentioned at the previous points.

The characterization of the XOU prototype at Panter will need an extension of the X-ray facility. It is foreseen that in a timeframe of about 1 year it shall be extended to be able to fully illuminate optics with focal lengths of the order of 20 $\mathrm{m}$ (like for IXO) and to measure in-focus images. The tests on the XOU prototype will be done a) in monochromatic setup at energies from 1 to $10 \mathrm{keV}$. Above this energy up to $50 \mathrm{keV} \mathrm{b}$ ) in energy-dispersive setup, consisting of a continuum Bremsstrahlung spectrum emitted by a $\mathrm{W}$-anode X-ray tube. The detectors that will be used are the pn-EPIC and TRoPIC CCDs, both endowed with a very good spatial (150 and $75 \mu \mathrm{m}$ pixel size respectively) and energy 
resolution $(\sim 0.2 \mathrm{keV}$ at $6 \mathrm{keV})$, then suitable for the measurements being planned. The focal spot recorded by the detector conveys all the information concerning the optical quality (i.e: angular resolution, with particular regard to the scattering off reflecting surfaces) and the effective area. Due to the finite distance of the source, the X-rays will impinge the mirrors with a non negligible divergence, that will be accounted for in the data reduction and analysis.

\section{CONCLUSION}

The development of a representative scaled module prototype for IXO is under study with the support of ESA. This study, acting as a risk mitigation measure to the Silicon Pore optics pursued by ESA, concerns the technology of the slumping of glass foils for the manufacturing of pair plates having Wolter I geometry. The investigation will study also the integration of these PPs in a XOU structure, preserving the individual shape of the PP and aligning together the PPs.

In a first phase of the study it will be individuated the best slumping technique (direct versus indirect) comparing the results in terms of mirror plate shape error and microroughness. The alignment and integration of the PPs in the XOU will be done using an Integration Machine having an optical feedback in the focal plane so to over impose with high accuracy the focal spots generated from the single PPs.

It is foreseen to measure the resulting XOU prototype, composed by 3 real PPs plus 17 dummy plates, in X-Rays at the Panter facility. This facility will be upgraded in the next year for the measurement of long focal length optics like that of IXO (20 m).

This study and the manufacturing of the XOU prototype is expected to last for 16 months.

\section{REFERENCES}

[RD1] M. J. Collon; S. Kraft; R. Günther; E. Maddox; M. Beijersbergen; M. Bavdaz; D. Lumb; K. Wallace; M. Krumrey; L. Cibik; M. Freyberg, "Performance characterization of silicon pore optics", Proc. SPIE, v. 6266, (2006)

[RD2] W. Zhang, "Lightweight X-ray Mirrors for the Constellation-X Mission", Proc. SPIE, 6266-68, 2006.

[RD3] W. Zhang, D. A. Content, J. P. Lehan, R. Petre, T. T. Saha, M. Gubarev, W. D. Jones, \& S. L. O’Dell, "Development of Lightweight X-ray Mirrors for the Constellation-X Mission", Proc. SPIE, 5900, 59000V

[RD4] Scott M. Owens, et al., "Alignment and test of a Constellation-X SXT mirror segment pair", Proc. SPIE Vol. 6266, (2006)

[RD5] P. Friedrich, et al., "Manufacturing of Wolter-I mirror segments with slumped glass", Proc. SPIE Vol. 6266, (2006)

[RD6] M. Vongehr et al., "Development of slumped glass mirror segments for large X-ray telescopes", Proc. SPIE, Vol. 7011, (2008)

[RD7] P. Friedrich, et al., "Recent results on manufacturing of segmented x-ray mirrors with slumped glass", Proc. SPIE Vol. 5900, (2005)

[RD8] M. Ghigo, G. Pareschi et al., "Slumped glass option for making XEUS mirrors: preliminary design and ongoing developments", Proc. SPIE Vol. 7011, (2008)

[RD9] M. Ghigo, G. Pareschi et al., "Development of active/adaptive lightweight optics for the next generation of telescopes", Proc. SPIE Vol. 6148, (2006)

[RD10] M. Ghigo, G. Pareschi et al., "Manufacturing of lightweight glass segments for adaptive optics", Proc. SPIE Vol. 6272, (2006)

[RD11] M. Ghigo, G. Pareschi et al., "Development of lightweight optical segments for adaptive optics", Proc. SPIE Vol. 6691, (2007)

[RD12] J. Schneider, "Vibration testing of the constellation $\mathrm{x}$ spectroscopy $\mathrm{x}$-ray telescope reflector mounting design", Aeronautical Engineering, June 2004 